\title{
Author Index Vol. 21, 1994
}

Supplement issue No. 1 has its own Author Index

Aihara, N. 184 Alexander, E. 2 Alonso-Vanegas, M. 192 Alvarez,J.L. 192 Antikainen, T. 77 Azzarelli, B. 232

Becker,|L.E. 91 Berger, M.S. 165,254 Black,|P.McL. 23 Boaz, J.C. 232 Boop, F.A. 237

Caldemeyer, K.S. 232 Canady, A.I. 201 Chapman, P.H. 179 Cheek, W.R. 227 Cheeseman, F. 71 Chou, J. 174 Ciquinin, O., Jr. 50 Cobb, M.A. 237 Cochrane, D.D. 59 Cordelia, L. 243 Cosman, E.R. 179 Cotcamp, D.H. 221 Crone, K.R. 221

Delgado, L. 192 Dhellemmes, P. 260 Domingo, Z. 45 Donahue,|DJ. 105 Drake,|J.M. 6 Duhaime, A.-C. 117

Elisevich, K. 71

Frenkel, E.P. 16

Galassi, E. 243 Geyer, J.R. 165,254 
Gibbons, P.A. 221 Godano, U. 243 Gonzalez-Gomez, I. 113 Greene, K.A. 124

Hamilton, M.G 124 Hendrick, E.B. 91 Henner, W.D. 16 Herbaux, B. 260 Herman, J.M. 124 Hladky, J.P. 260 Hoffman,|H.J. 91,104 Holmes, G. 23 Hornyik, G. 189 Humphreys, R.P. 44,91 , 178 184 Ishikawa, T. Ito, J. 36

James, H.E. 65 Jimenez, J.L. 192 Johnson, D.L. 83,90

Kahana, M.D. 221 Kallioinen, M. 77 Kamiya, K. 184 Kanayama, M. 184 Katano, H. 184 Kaufman, B.A. 36 Kaufman, R.A. 105 Kestle, J.R.W. 59 Klosterman,|A. 36 Kupsky, W|J. 23

Laham, J.L. 221 Laurent, J.P. 227 Lecomte-Houcke, M. 260 Lee, M. 174 Lejeune, J.P. 260 Leong, T. 23 
McComb, J.G. 113 McCullough, D.C. 83 Manreza, L.A. 50 Marciano, F.F. 124 Marino, R., Jr. 50 Martino, A.M. 201 Mattar, A.G. 71 Medlock, M, D. 113 Mendizabal, R. 192 Mickell, JJ. 55 Mickey, B.E. 16 Milhorat, T.H. 112 Miller, J.I. 112 Molleston, M.C. 195 Moran, C.J. 195 Moura dos Santos, A.L. 50 Muhlbauer, M.S. 105 Muszynski, C.A. 227 Myseros, J.S. 55

Nagai, H. 184 Nagib, M.G. 31 Neuwelt, E.A. 16

Park, T.S. 36 Pereira Plese, J.P. 50 Peter,J.C. 45 Piatt, J.H., Jr. 66,189 Pohjonen, T. 77 Poskitt, K.J. 59 Pozzati, E. 243

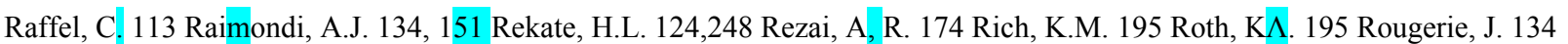


Sanchez-Cabrera, J.M. 192 Sanford, R.A. 105 Scott, R.M. 1,23 Seng Shu, E.B. 50 Serlo, W. 77 Shady, J.A. 23 Singer, B. 260 Sivalsganathan, S. 6 Smith, R.R. 232 Smoker, W.R.K. 55 Sood, S. 201 Spetzler, R.F. 124 Steinbok, P. 59

Tarbell, NJ. 23 Tenti, G. 6 Terbrock, A. 36 Törmälä, P. 77 Trumble, E.R. 55

Villiers, J.C. de 45

Wang, E.C. 165 Ward, J.D. 55 Waris, T. 77 Warner,W.C. 105 West, G.A. 254 Wiliams, P.C. 16 Winters, N. 36

Yamashita, N. 184 Yoshida, M. 91

Zervas, N.T. 179 